University of Nebraska - Lincoln

DigitalCommons@University of Nebraska - Lincoln

April 1996

\title{
Magnetic viscosity and switching volumes of annealed Fe/Pt multilayers
}

C.P. Luo

University of Nebraska - Lincoln

Z.S. Shan

University of Nebraska - Lincoln

David J. Sellmyer

University of Nebraska-Lincoln, dsellmyer@unl.edu

Follow this and additional works at: https://digitalcommons.unl.edu/physicssellmyer

Part of the Physics Commons

Luo, C.P.; Shan, Z.S.; and Sellmyer, David J., "Magnetic viscosity and switching volumes of annealed Fe/Pt multilayers" (1996). David Sellmyer Publications. 89.

https://digitalcommons.unl.edu/physicssellmyer/89

This Article is brought to you for free and open access by the Research Papers in Physics and Astronomy at DigitalCommons@University of Nebraska - Lincoln. It has been accepted for inclusion in David Sellmyer Publications by an authorized administrator of DigitalCommons@University of Nebraska - Lincoln. 


\title{
Magnetic viscosity and switching volumes of annealed Fe/Pt multilayers
}

\author{
C. P. Luo, Z. S. Shan, and D. J. Sellmyer \\ Behlen Laboratory of Physics and Center for Materials Research and Analysis, University of Nebraska, \\ Lincoln, Nebraska 68588-0113
}

\begin{abstract}
Magnetic viscosity and the phenomenon of the sweep-rate dependence of the coercivity are important for the characterization of magnetic recording media. The activation volume of magnetization reversal or switching volume is directly related to medium noise. The magnetic viscosity behavior of annealed Fe/Pt multilayers was studied and the switching volumes were obtained by both measurements of the sweep-rate dependence of coercivity and time dependence of magnetization. It is found that samples with larger coercivities have smaller switching volumes, and an estimate of the magnetic grain diameter is about $9 \mathrm{~nm}$. The coercivity mechanisms are also discussed. (C) 1996 American Institute of Physics. [S0021-8979(96)07808-8]
\end{abstract}

\section{INTRODUCTION}

Magnetic viscosity parameters are directly associated with the microscopic structure of materials and with the processes responsible for magnetization. The analysis of magnetic viscosity may allow a better understanding of magnetization reversal and coercivity mechanisms. For magnetic recording media, the activation volume of reversal or switching volume is an essential parameter which is related to the media noise. Previously, we have reported that annealed $\mathrm{Fe} / \mathrm{Pt}$ multilayers with fct structure have large in-plane coercivities and fine grain sizes, ${ }^{1}$ which suggests potential applications in future ultrahigh-density magnetic recording. In this work, we studied the magnetic viscosity properties and the switching volumes of the annealed Fe/Pt multilayers.

\section{MAGNETIC VISCOSITY}

The phenomenon of magnetic viscosity has its origins in thermal agitation of magnetic moment over energy barriers. In many materials it is found experimentally that

$$
M(H, t)=M\left(H, t_{0}\right)-S(H) \ln (t),
$$

where $S(H)$ is the viscosity coefficient. According to Street and Woolley, ${ }^{2}$

$$
S(H)=\chi_{\mathrm{irr}}(H) H_{f}(H),
$$

where $\chi_{\text {irr }}$ is the irreversible susceptibility characterizing the barrier hopping and $H_{f}$ the fluctuation field which is defined by

$$
H_{f}(H)=k_{B} T /\left[V^{*}(H) M_{s}\right]
$$

where $V^{*}(H)$ is the activation volume of reversal, $k_{B} T$ the Boltzmann energy, and $M_{s}$ the magnetic moment per unit volume.

Another aspect of the time-dependent behavior is the sweep-rate $\left(d H_{a} / d t\right)$ dependence of the coercivity. Higher sweep-rate measurements generally give larger values of $H_{c}$ than lower sweep-rate measurements. Under certain assumptions, the coercivity varies linearly with respect to $\ln \left(d H_{a} / d t\right):^{3}$

$$
H_{c}=k_{B} T /\left(V^{*} M_{s}\right)\left[\ln \left(d H_{a} / d t\right)+\text { const }\right] .
$$

The activation volume $V^{*}$ can be obtained from the slope of the $H_{c} \sim \ln \left(d H_{a} / d t\right)$ plot.

\section{SAMPLES AND MEASUREMENTS}

$\mathrm{Fe} / \mathrm{Pt}$ multilayers were deposited by $\mathrm{rf}$ and dc sputtering on cover glasses and annealed at $300{ }^{\circ} \mathrm{C}$ for $30 \mathrm{~min}$. The base pressure of the sputtering system was $2 \times 10^{-7}$ Torr and the Ar pressure was 5 mTorr during deposition. All magnetic measurements were made using a Micromag-2900 alternating gradient force magnetometer. For each measurement of the time dependence of magnetization, the sample was initially saturated with a positive field of $10 \mathrm{kOe}$, and then a constant negative test field was applied. In order to obtain the $\chi_{\text {irr }}(H)$ curves, the de demagnetization (DCD) and the isothermal remanence magnetization (IRM) curves were measured.

\section{RESULTS AND DISCUSSIONS}

\section{A. Magnetic parameters}

The hysteresis loops of the annealed $\mathrm{Fe} / \mathrm{Pt}$ multilayers with different bilayer thickness $\left(d_{b}\right)$ were measured with applied field in the film plane. All of the measured samples had the same composition and a total thickness of $150 \AA$. The magnetic parameters such as coercivity $\left(H_{c}\right)$ and squareness $\left(M_{r} / M_{s}\right)$ are listed in Table I. Rather square hysteresis loops have been obtained for all samples. The table also shows that the coercivities are strongly dependent on $d_{b}$, which will be discussed later.

Figure 1 shows the $\chi_{\text {irr }}(H)$ curves of the examined samples, which were obtained by differentiating the DCD and IRM curves, respectively. According to $\mathrm{O}^{\prime} \mathrm{Grady}^{4}$ and Donnet et al., ${ }^{5}$ the IRM differential $\left(\chi_{\text {irr }}^{i}\right)$ measures the distribution of energy barriers to wall motion; while the DCD differential $\left(\chi_{\text {irr }}^{d}\right)$ measures the energy barrier to domain nucleation in the first instance and then examines the mechanism by which reversal proceeds. Thus, both quantities provide direct information about magnetization reversal, and their relations to coercivity and activation volume will be discussed later.

\section{B. Magnetic viscosity properties}

\section{Sweep rate dependence of $\boldsymbol{H}_{c}$}

Measurements of the coercivities as a function of sweep rates were carried out for samples A, B, C, and D with sweep rates varying from 10 to $1000 \mathrm{Oe} / \mathrm{s}$. The results shown in 
TABLE I. The magnetic parameters and switching volumes of the annealed Fe/Pt multilayers.

\begin{tabular}{lccccc}
\hline \multicolumn{1}{c}{ Samples $^{\mathrm{a}}$} & $\mathrm{A}$ & $\mathrm{B}$ & $\mathrm{C}$ & $\mathrm{D}$ & $\mathrm{E}$ \\
\hline$d_{b}(\AA)$ & 15 & 30 & 37.5 & 50 & 75 \\
$H_{c}(\mathrm{Oe})^{\mathrm{b}}$ & 934 & 2220 & 2560 & 3140 & 1140 \\
$M_{r} / M_{s}$ & 0.85 & 0.89 & 0.87 & 0.87 & 0.85 \\
$V^{*}\left(\times 10^{-18} \mathrm{~cm}^{3}\right)^{\mathrm{c}}$ & 6.96 & 1.42 & 1.93 & 1.20 & $\cdots$ \\
$V^{*}\left(H_{c}\right)\left(\times 10^{-18} \mathrm{~cm}^{3}\right)$ & 2.03 & 1.51 & 1.33 & 1.25 & 2.42 \\
\hline \hline
\end{tabular}

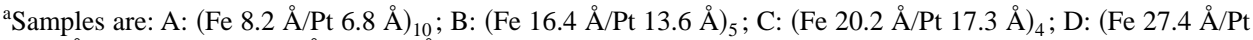

$22.6 \AA)_{3}$, and $\mathrm{E}:(\mathrm{Fe} 40.4 \AA / \mathrm{Pt} 34.6 \AA)_{2}$. All samples are the same total thickness and were annealed at $300{ }^{\circ} \mathrm{C}$

for $30 \mathrm{~min}$.

${ }^{\mathrm{b}} H_{c}$ was measured at a sweep rate of 250 Oe/s.

${ }^{\mathrm{c}} V^{*}$ was determined from the measurement of the sweep-rate dependence of coercivity.

${ }^{\mathrm{d}} V^{*}\left(H_{c}\right)$ is the switching volume when the applied field equals to $H_{c}$.

Fig. 2 are that $H_{c}$ linearly depends on $\ln \left(d H_{a} / d t\right)$. So Eq. (4) can be applied to analyze the results and the switching volumes are obtained, as listed in Table I. A small switching volume of $1.2 \times 10^{-18} \mathrm{~cm}^{3}$ has been measured for sample D which has a coercivity of $3.14 \mathrm{kOe}$. As the total film thickness $h$ is $150 \AA$, the magnetic grain size which is defined as $\left(V^{*} / h\right)^{1 / 2}$ is about $90 \AA$.

\section{Time dependence of magnetization}

Logarithmic time decays have been observed for all samples under constant negative applied fields. As an ex-

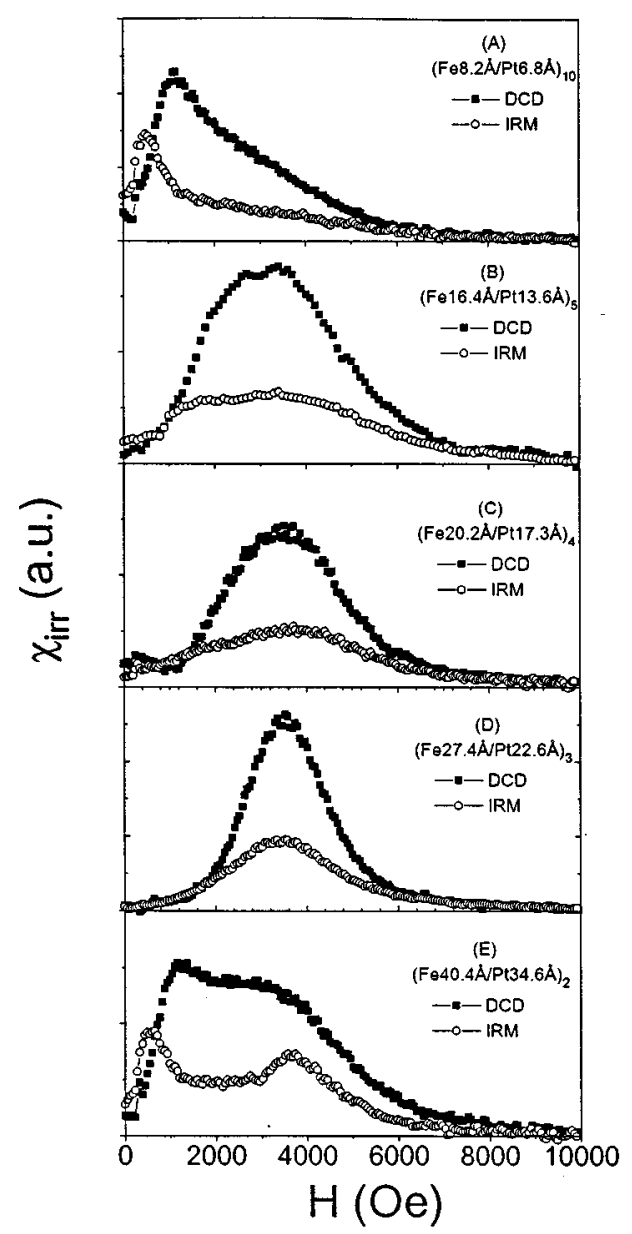

FIG. 1. Differentiated remanence curves for annealed Fe/Pt multilayers. ample, Fig. 3 shows the magnetization varying linearly with $\ln (t)$ for sample $\mathrm{C}$ under different applied fields. From these plots the viscosity coefficients $S(H)$ were obtained, as shown in Fig. 4. The variation of $S$ with $H$ essentially mirrors the behavior of $\chi_{\text {irr }}(H)$ which gives a measure of irreversible processes in the film.

From $S(H)$ and $\chi_{\mathrm{irr}}(H), V^{*}(H)$ can be obtained, as shown in Fig. 5. Clearly, $V^{*}(H)$ is not a constant. It depends on the applied fields. This arises from the fact that the applied field may affect both the height and the distribution of energy barriers. In the low-field region, both $\mathrm{A}$ and $\mathrm{E}$ have much larger $V^{*}$ than the other samples, while in the highfield region, all samples tend to have the same $V^{*}$. At each sample's coercive field, the sample with larger $H_{c}$ shows smaller $V^{*}\left(H_{c}\right)$. The values of $V^{*}\left(H_{c}\right)$ are matched very well with $V^{*}$ obtained from the sweep-rate dependence measurement of coercivity except for sample A, for which $V^{*}$ is a little larger than $V^{*}\left(H_{c}\right)$.

Both $H_{c}$ and $V^{*}$ are related to the magnetization reversal mechanisms which depend on the microstructure. Figure 1 reflects how the distribution of energy barriers varies with $d_{b}$. In the low-field region, for samples A and E, the nucleation field is greater than the domain-wall-pinning field as can be seen from the $\chi_{\mathrm{irr}}(H)$ curves. Hence, following the

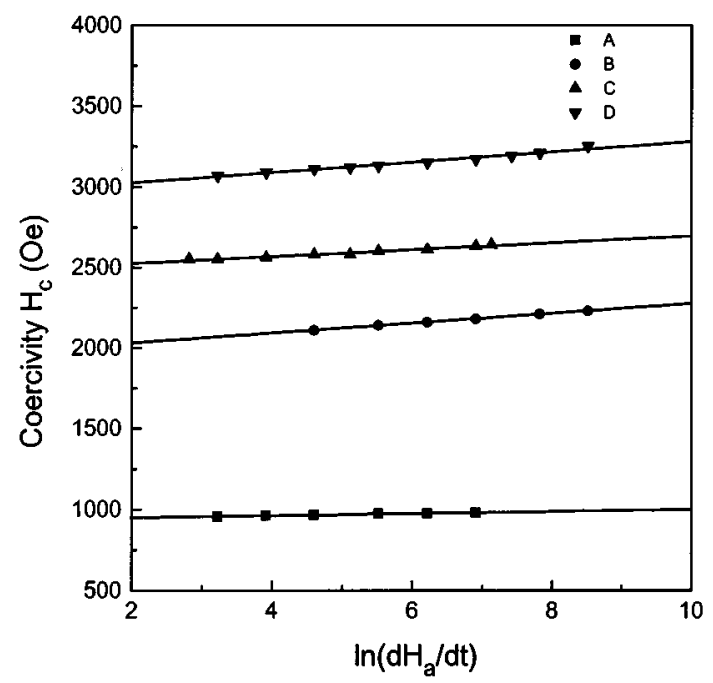

FIG. 2. The variation of $H_{c}$ with sweep rate $d H_{a} / d t$. 


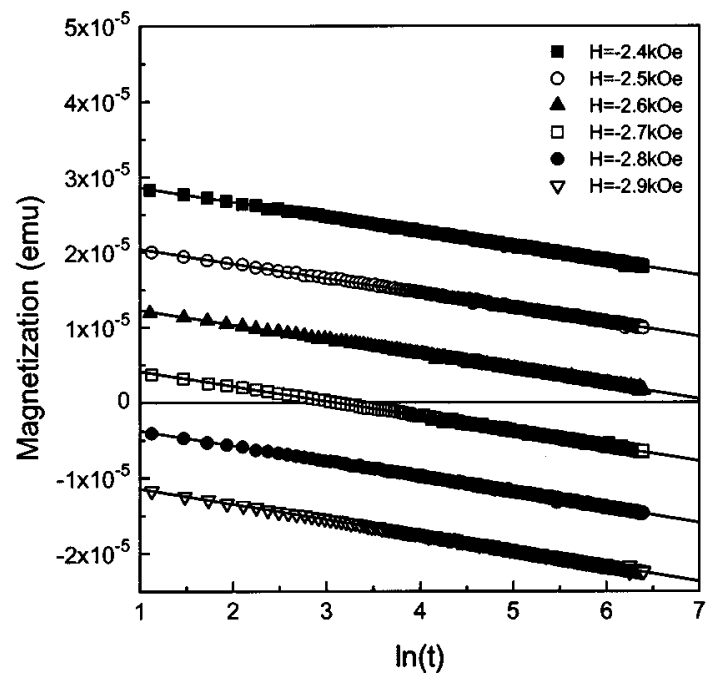

FIG. 3. Time decay of magnetization in the presence of constant magnetic fields in the direction opposite to the initial magnetization for sample C.

analysis of O'Grady, ${ }^{4}$ once the domain is nucleated it expands quickly, causing a sharp increase in switching volumes. And then the decrease of activation volume with applied field may be associated with the pinning of the movement of the nucleated domain walls.

The coercivity is also related to the exchange coupling between grains. Generally the coercivity increases with the reduction of exchange coupling. ${ }^{6}$ For a system without interaction, $\chi_{\text {irr }}^{d}$ and $\chi_{\text {irr }}^{i}$ should be identical with $\chi_{\text {irr }}^{d}=2 \chi_{\text {irr }}^{i}$. This suggests that the grains are more strongly exchange coupled for samples $\mathrm{A}$ and $\mathrm{E}$, for which the $\chi_{\text {irr }}^{i}$ curves increase at lower field than the $\chi_{\text {irr }}^{d}$ curves. ${ }^{7}$ The exchange coupling ap-

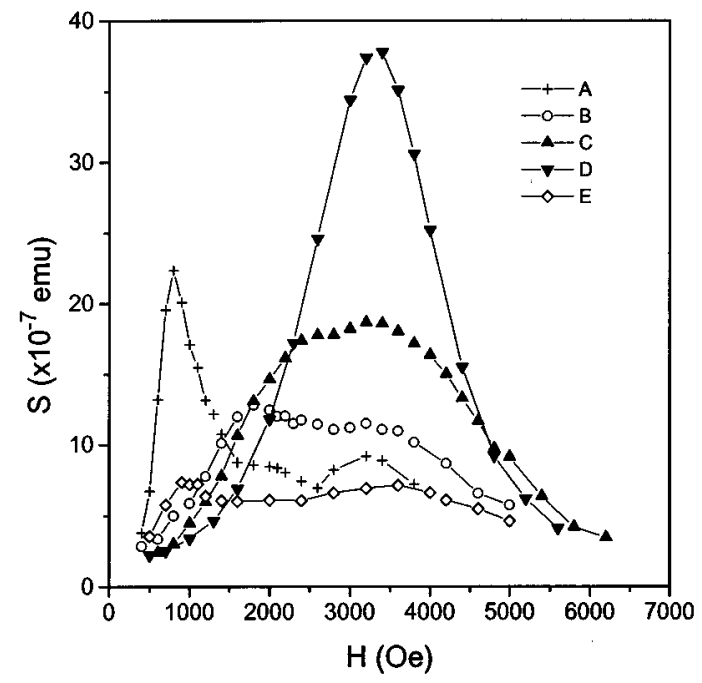

FIG. 4. The variation of viscosity coefficient $S$ with applied field $H$.

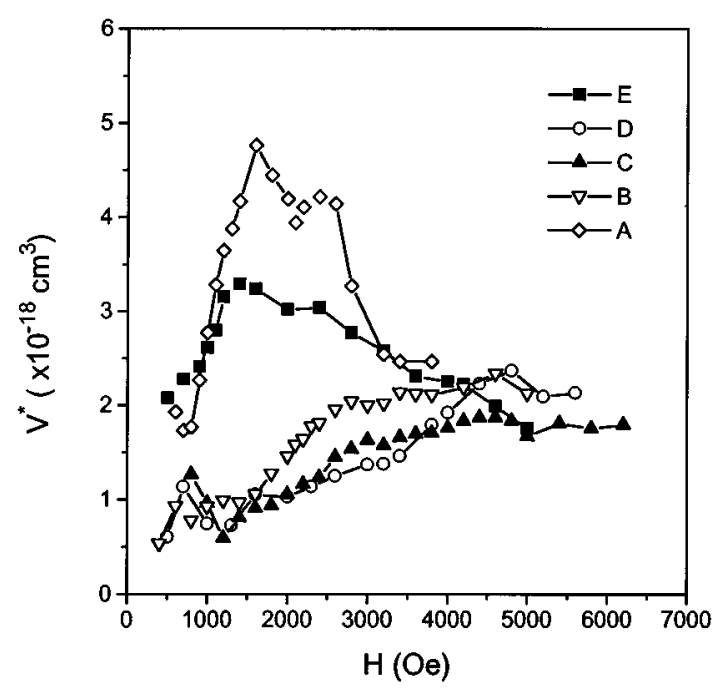

FIG. 5. The variation of activation volumes with applied field.

pears to be reduced for samples B, C, and D as the $\chi_{\text {irr }}^{i}(H)$ and $\chi_{\text {irr }}^{d}(H)$ curves are centered at the same position. Highresolution transmission electron microscopy (TEM) data on these samples show grain sizes of about $15 \mathrm{~nm}$. The large value of $H_{c}$ in sample $\mathrm{D}$ presumably is connected with a smaller exchange coupling, but the correlation of this with details of the nanostructures awaits further TEM studies.

\section{SUMMARY}

Through the study of magnetic viscosity behavior of the annealed Fe/Pt multilayers, their activation volumes of reversal were obtained. Films with larger coercivities have smaller switching volumes. Given the small estimated value of the magnetic grain size $(\sim 90 \AA)$ and the large and controllable coercivities, these films may have potential as new ultrahigh-density recording media.

\section{ACKNOWLEDGMENTS}

This research was supported by NSF under Grant No. DMR-9222976 and by the CMRA.

${ }^{1}$ C. P. Luo and D. J. Sellmyer, IEEE Trans. Magn. 31, 2764 (1995).

${ }^{2}$ R. Street and J. C. Woolley, Proc. Phys. Soc. London Sect. A 62, 562 (1949).

${ }^{3}$ P. Bruno, G. Bayreuther, P. Beauvillain, C. Chappert, G. Lugert, D. Renard, J. P. Renard, and J. Seiden, J. Appl. Phys. 68, 5759 (1990).

${ }^{4}$ K. O'Grady, T. Thomson, and S. J. Greaves, J. Appl. Phys. 75, 6849 (1994).

${ }^{5}$ D. M. Donnet, V. G. Lewis, J. N. Chapmant, K. O'Grady, and H. W. van Kesteren, J. Phys. D 26, 1741 (1993).

${ }^{6}$ I. L. Sanders, T. Yogi, J. K. Howard, S. E. Lambert, G. I. Gorman, and C. Hwang, IEEE Trans. Magn. MAG-25, 3869 (1989).

${ }^{7}$ K. O'Grady, R. W. Chantrell, and I. L. Sanders, IEEE Trans. Magn. 29, 286 (1993). 